\title{
Elements of Fractal Generalization of Dual-Porosity Model for Solute Transport in Unsaturated Fractured Rocks
}

\author{
Leonid Bolshov*, Peter Kondratenko*, Leonid Matveev*, and Karsten Pruess** \\ *Russian Academy of Sciences, \\ Nuclear Safety Institute, 52 Bolshaya Tul'skaya St., 115191 Moscow, Russia; \\ ** Earth Sciences Division, Lawrence Berkeley National Laboratory. Berkeley, CA 94720
}

In this study, new elements were developed to generalize the dual-porosity model for moisture infiltration on and solute transport in unsaturated rocks, taking into account fractal aspects of the percolation process. Random advection was considered as a basic mechanism of solute transport in self-similar fracture systems. In addition to spatial variations in the infiltration velocity field, temporal fluctuations were also taken into account. The rock matrix, a low-permeability component of the heterogeneous geologic medium, acts as a trap for solute particles and moisture. Scaling relations were derived for the moisture infiltration flux, the velocity correlation length, the average velocity of infiltration, and the velocity correlation function. The effect of temporal variations in precipitation intensity on the infiltration processes was analyzed. It showed that the mode of solute transport is determined by the power exponent in the advection velocity correlation function and the dimensionality of the trapping system, both of which may change with time. Therefore, depending on time, various transport regimes may be realized: superdiffusion, subdiffusion, or classical diffusion. The complex structure of breakthrough curves from changes in the transport regimes was also examined. A renormalization of the solute source strength due to characteristic fluctuations of highly disordered media was established.

The "dual-porosity" concept (Barenblatt et al., 1960; Gerke and van Genuchten, 1993; Pruess, 1999; Ray et al., 1997) is the best-known theoretical model for describing moisture infiltration and solute transport processes in fractured media. It is based on approximating a geologic medium with a superposition of two overlapping homogeneous subsystems, both of which occupy the entire volume under consideration and have their own hydrologic and transport properties and porosity. One of these subsystems, $f$, represents the fracture system, and the other one the rock matrix, $m$. In this description, the geologic medium is characterized by two sets of parameters for the velocity, fluid pressure, moisture content, and solute concentration that are functions of spatial coordinates and time.

The governing equations for moisture infiltration are written in the form of two massbalance conditions involving Darcy's flux law for each subsystem. Constitutive relations between partial pressure heads and saturations are required to close the set of governing equations. Similarly, the equations describing solute transport dynamics are written as mass conservation laws, with the use of Fick's law for the solute fluxes. Both sets of equations take into account the interaction between the fracture system and the matrix, which entails both moisture and solute exchange.

The classical dual-porosity model can take into account strong heterogeneity in the geologic medium with sharp property contrasts between domains. At the same time, the model is based on an implicit assumption of statistical homogeneity. We present arguments (see a review in Bolshov et al., 2008), however, to show that for unsaturated fractured rocks in the vicinity of the percolation threshold, the structure of percolation channels has fractal properties. In such systems, Darcy's law and the usual advection-diffusion model (when the solute flux is determined by Fick's law with consideration of drift) are not suitable to describe the main characteristics of the percolation flux and solute transport. A first attempt at modeling dual-porosity systems as percolation networks was presented by Chang and Yortsos (1990), who described the averaged characteristics of single-phase, slightly compressed fluid flow in a fractal object by means of an appropriate modification of the diffusivity equation. Scaling behavior of the unsaturated hydraulic conductivity in porous media at the dry end of the moisture content was studied by Hunt (2004a,b,c) within the framework of continuum percolation theory.

Our objective was to develop elements of a generalized dual-porosity model for moisture 
infiltration and solute transport in unsaturated fractured rocks, taking into account fractal aspects of the percolation process. These elements include random solute advection due to moisture infiltration in percolation networks of channels, and interaction between "fast" (fractures) and "slow" (matrix) subsystems that take into account their fractal properties. In pursuing this objective, we took advantage of recent progress in theories of percolation and critical phenomena (ben-Avraham and Havlin, 2000; Isichenko, 1992; Ma, 1976).

\section{Fractal Properties of Flow for Moisture Infiltration in Unsaturated Fractured Rocks}

It is well known from studies of seepage in porous media (Dullien, 1979) that some amount of moisture always remains in the medium during drainage (irreducible or residual saturation). At low saturation, the moisture accumulates in the narrowest sections of cracks and is retained there in droplets held by capillary forces. Additional moisture supply causes the droplets to grow and, after reaching some critical size, the droplets become unstable and start moving. In Hunt (2004c) this motion of moisture at the dry-end moisture content was considered as film flow. During the motion, it may break into smaller droplets, which may become trapped into new positions, and then the process repeats. As a result, many droplets are involved in the motion and the process has a self-organized, collective nature. Such a dynamic system (the set of moving interacting droplets) belongs to the class of extended dissipative dynamic systems. The existence of many metastable states (various configurations of "hanging" droplet sets) makes the system pass from one metastable state to another. In the present work, we considered the dynamics of such a system using the concept of self-organized criticality (SOC) (Bak et al., 1987). Although a comprehensive description of SOC processes is still unavailable, there are three robust features of the SOC state that are important for our model (Bak et al., 1987, 1988; Vespignani and Zapperi, 1998):

1. Metastable dynamic structures have spatial self-similarity. This means that the set of droplet trajectories (fractures through which moisture flows) can be regarded as a fractal cluster strongly resembling a percolation cluster. Based on this property, we used the results of percolation theory for the description of stationary (time-averaged) infiltration flow (Dykhne, 2004a).

2. Flicker noise (or $1 / f$ noise), characterized by correlations extending across a wide range of time scales, has a frequency spectrum $\sim 1 / f^{\alpha}$ with $\alpha \sim 1$. This means that along with spatial self-similarity, temporal self-similarity should also be taken into account when deriving scaling relations, and hence that the time variable should have its own scale dimension (see below).

3. Criticality arises from the fine tuning to zero of one or more control parameters (driving rate and dissipation). In the case of infiltration, the external flux is such a parameter. The system possessing the property of SOC naturally evolves to the final state regardless of the initial conditions (i.e., the critical state is an attractor of the dynamics).

Moreover, the SOC state is robust with respect to variations in microscopic parameters. This means that the processes of droplet formation, retention in fracture tips, motion, breaking, and junc- tion do not influence the principal characteristics of the processes described in Items 1 and 2.

It should be stressed that formation of self-similar metastable dynamic structures does not require the fracture or pore network to be fractal. If the network happens to be fractal, however, this will not change the situation because it is also random.

Below we suppose that conditions necessary for bringing the system into the SOC state are fulfilled, and that flow properties and hence the solute transport are determined by Items 1 and 2 above.

\section{Scaling Relations for Moisture Infiltration Taking into Account the Percolation Structure of the Media}

In accordance with Item 1 above, flow properties in unsaturated fractured rocks are determined by the percolation structure of the infiltration cluster. According to modern concepts, percolation systems are closely related to condensed media near a phase transition of the second kind (ben-Avraham and Havlin, 2000).

The most essential feature of macroscopic systems undergoing second-kind phase transition is the presence of large-scale fluctuation of the order parameter (e.g., magnetization in the vicinity of the transition temperature into the ferromagnetic state). As a consequence of such behavior, correlations are powerlike functions of spatial coordinates. Quite similar regularities arise in the functional dependence of mean physical quantities on proximity to the phase transition point. It is important to note that power exponents in all these dependencies are universal, being determined by general symmetry properties of the systems, independent of the details of interatomic interactions. The presence of large-scale clusters in percolation media makes them similar to condensed systems in the vicinity of the phase transition point.

Fluctuations of the order parameters, whose correlation lengths tend to infinity at the critical point, are the analogs of clusters in percolation media. Specifically, fractal properties (in other words, scale-invariance properties) of clusters and of fluctuations of the order parameter provide an opportunity to describe phenomena near the percolation threshold as well as near the critical point by scaling relations. These relations have the form of power dependencies on the threshold proximity parameter.

For near-critical systems, this parameter is a temperature deviation from the critical point, while for percolation systems it is the deviation of the average number of active (connected) structural elements from the threshold value, $p-p_{\mathrm{cr}}$. For unsaturated media, a measure of threshold proximity may be expressed with the parameter $\Delta S=S-S_{\text {cr }}$ (see Dykhne, 2004a), where $S$ is moisture saturation and $S_{\mathrm{cr}}$ is its residual value.

Thus, for infiltration processes in unsaturated fracture systems above the percolation threshold, the scaling relations in the infinite volume limit take the form

$\xi \propto \Delta S^{-v}$

$u \propto \Delta S^{\mu}$

where $\xi$ is the spatial extent of conductive fracture clusters (see Dykhne, 2004a), $u$ is the average volumetric flux of moisture (the same as infiltration rate), and $\nu$ and $\mu$ are scaling exponents. Note 
that for saturated media, relations of the type of Eq. [1] and [2] with $\Delta p$ instead of $\Delta S$ have been suggested by Berkowitz and Braester (1991) and Sahimi and Imdakh (1998).

Our objective now is to consider correlation properties of the seepage velocity and to generalize the percolation theory relations to the case of finite systems. From this point of view, instead of $\Delta S$, the correlation length $\xi$ should be regarded as a more suitable and natural variable for representing the proximity of the system to the percolation threshold.

Using Eq. [1] to express $\Delta S$ in terms of the correlation length $\xi$ and substituting into Eq. [2], we obtain

$u=V\left(\frac{a}{\xi}\right)^{b}$

where $a$ is the lower limit of the interval for which fractal properties hold. While the variable $u$ by its nature is a large-scale one (i.e., characterizing the system at scales of order $\xi$ or larger), the parameter $V$ is determined by local medium properties at scales on the order of the lower limit of the fractality interval $a$, and describes the flux density or velocity amplitude at these scales. The new power exponent is related to the previous one by

$h=\mu / \nu$

The local value of the moisture infiltration velocity can be represented in the form of a sum: $\mathbf{v}(\mathbf{r})=\mathbf{u}+\mathbf{v}^{\prime}(\mathbf{r})$, where $\mathbf{u}$ describes the average flow rate and $\mathbf{v}^{\prime}(\mathbf{r})$ is a random component fluctuating in space. An important quantity describing aspects of random flow is the pair velocity correlation function. This function is determined by the product of velocities at two different points, averaged across an ensemble of realizations. Within the framework of the theory of critical phenomena (Ma, 1976; see also below), it can be shown that the quantities $\mathbf{u}$ and $\mathbf{v}^{\prime}(\mathbf{r})$ should have the same scaling dimensions because they are components of the infiltration velocity. Using the fact that $\mathbf{r}$ and $\xi$ also have the same scale dimension, it follows from Eq. [3] that, at $a<<$ $r<<$,

$\left\langle v_{i}^{\prime}(\mathbf{r}) v_{j}^{\prime}(0)\right\rangle \sim V^{2}\left(\frac{a}{r}\right)^{2 b}$

where \langle\rangle denotes ensemble averaging and $r=|\mathbf{r}|$. When $r\rangle \xi$, the correlation function decays exponentially.

Equations [3] and [5] are valid under the condition that the size of the medium, $L$, is much greater than the correlation length: $L>>$; however, these relations remain at least qualitatively true under the condition $L \sim \xi$ (see Sahimi, 1993, for more rigorous derivation). Hence, above the percolation threshold, the correlation length can be related to the flux density by

$\xi=L\left(\frac{u_{1}}{u}\right)^{1 / h}$

where the size-dependent variable $u_{1}$ is determined by

$u_{1}(L)=V\left(\frac{a}{L}\right)^{\bar{\mu}}$

Since the average flux density $u$ is in fact the control parameter determining the state of the system, Eq. [6] can be regarded as the relation determining the dependence of the correlation length $\xi$ of the stationary infiltration pattern on $u$. For $u>u_{1}$, we have $\xi$ $<L$. When $u<u_{1}$, moisture flow becomes nonstationary.

Note that near the percolation threshold we have

$a<<\xi, L$

Therefore, two important consequences arise from Eq. [3] and [5]. The first one is the inequality

$V>>u$

The second consequence consists of the fact that the random component of the velocity $\mathbf{v}^{\prime}$ becomes comparable with the average velocity $u$ at scales $\min (\xi, L)$ but is greater than $u$ at smaller scales.

\section{Dynamic Fluctuations in Moisture Infiltration}

Now let us consider the consequences of the dynamic fluctuations inherent to the SOC state, in accordance with Item 2 above. Due to these fluctuations, the pair velocity correlation function should depend not only on spatial coordinates but also on time:

$$
\begin{aligned}
& K_{i j}^{(2)}(\mathbf{r}, t)=\left\langle v_{i}^{\prime}\left(\mathbf{r}_{1}, t_{1}\right) v_{j}^{\prime}\left(\mathbf{r}_{2}, t_{2}\right)\right\rangle ; \\
& \mathbf{r}=\mathbf{r}_{1}-\mathbf{r}_{2}, t=t_{1}-t_{2}
\end{aligned}
$$

Due to the self-similarity property in the region of fractality $r<$ $\xi$, the function $K_{i j}(\mathbf{r}, t)$ obeys the relation

$$
K_{i j}^{(2)}\left(\lambda \mathbf{r}, \lambda^{\Delta_{t}} t\right)=\lambda^{-2 h} K_{i j}(\mathbf{r}, t)
$$

where $\Delta_{t}$ and $h$ are the scaling dimensions of time and velocity, respectively. Index $h$ was introduced above in Eq. [3]. The index $\Delta_{t}$ is a new one. It characterizes dynamic properties of the system (Ma, 1976).

Consider the space-time Fourier transform of the pair velocity correlation function

$$
K_{i j}^{(2)}\{\mathbf{k}, \omega\}=\int d^{3} r \mathrm{~d} t K_{i j}^{(2)}(\mathbf{r}, t) \exp [-i(\mathbf{k r}-\omega t)]
$$

The most general form of this function is

$$
K_{i j}^{(2)}\{\mathbf{k}, \omega\}=K_{i j}^{(2)}\{\mathbf{k}\}\left[\delta(\omega)+\frac{1}{\omega} F(\omega, k)\right]
$$

with

$$
K_{i j}^{(2)}\{\mathbf{k}\} \propto V^{2} a^{2 h} k^{2 h-3} \quad \text { at } k a<<1
$$

and

$$
F(\omega, k)=f\left[\frac{\omega\left(\omega t_{1}\right)^{z-1}}{V a^{h} k^{1+h}}\right], \quad z=\frac{1+h}{\Delta_{t}}, \quad t_{1}=\frac{a}{V}
$$

Equation [13] expresses the fact that, due to the scaling relation Eq. [11] and definitions Eq. [12] and [14], the function $K_{i j}{ }^{(2)}$ $\{\mathbf{k}, \omega\}$ should be the product of $K_{i j}{ }^{(2)}\{\mathbf{k}\}$ and a quantity having the dimension of inverse frequency. The most general form of the latter corresponds to the expression in square brackets of Eq. [13], where the term proportional to $\delta(\omega)$ is the result of a 
Laplace transform of the stationary part of the correlation function of Eq. [10].

The first term on the right-hand side of Eq. [13] corresponds to a finite (nonzero) limit of the function $K_{i j}^{(2)}(\mathbf{r}, t)$ at $t \rightarrow \infty$. The second term describes dynamic fluctuations. The structure of the self-similar variable entering the function $f$ in Eq. [15] solely follows from the invariance of the correlation function under the scaling transformation in the range of fractality (see Eq. [11]). For this condition, the above structure can describe the dynamics of systems with different physical natures. Using this scaling relation below, we estimate the influence of time-dependent external conditions on the characteristics of the advection velocity field.

As is well known from electrodynamics, when a high-frequency electromagnetic field is applied to a massive conductor, the field only penetrates into a thin layer of the specimen, which is called the skin layer. With increasing frequency of the field, the skin layer becomes thinner. A similar skin effect holds for any classical diffusion process, such as propagation of land surface temperature variations (daily or seasonal) into the earth. An analogous situation arises in the distribution of the infiltrationvelocity field. Rapid variations in precipitation intensity can only persist in a thin layer near the infiltration boundary. At larger distances from the boundary, the medium perceives only the average value of the precipitation intensity.

Now we proceed to estimate the thickness $\delta$ of the infiltration boundary layer in dependence on the period $T$ of variations in the precipitation intensity. The relation between the characteristic values of the wave vector $\mathbf{k}$ corresponding to the skin-layer thickness and the frequency $\omega$ determining transient infiltration follows from the condition that the argument of the function $f$ in Eq. [15] be on the order of unity. From this we have

$\mathbf{k} \sim \frac{1}{a}\left(\frac{a \omega}{V}\right)^{z /(1+h)}$

Using $\mathbf{k} \sim \delta^{-1}$ and $\omega \sim T^{-1}$, we arrive at an estimate for the thickness $\delta$ of the top boundary layer as a function of the time period for variations in the precipitation activity:

$\delta \sim a\left(\frac{V T}{a}\right)^{\beta}, \quad \beta=\frac{z}{1+b}$

The parameter $V$ may be estimated as

$V \sim \frac{\kappa}{v} g$

where $\kappa$ is the local effective permeability, $v$ the kinematic viscosity of water, and $g$ the acceleration due to gravity. For conditions applicable to Yucca Mountain, Nevada, we take $a \sim\left(10^{-2}-10^{-1}\right)$ $\mathrm{m}, \kappa \sim 10^{-12} \mathrm{~m}^{2}$ (Pruess, 1999), $g \cong 10 \mathrm{~m} \mathrm{~s}^{-2}, v \cong 10^{-6} \mathrm{~m}^{2} \mathrm{~s}^{-1}$, $\beta \sim 1 / 2$, and $T \sim 1 \mathrm{yr} \sim 3 \times 10^{7}$ s. From Eq. [17] and [18] we then obtain the following estimate for the boundary layer thickness corresponding to seasonal precipitation variations:

$\delta_{s} \sim(2-5) \mathrm{m}$

Therefore, the seasonal variations lead only to a disturbance of the advection-velocity field in a subsurface layer whose thickness may be small compared with the thickness of the vadose zone (of order $L \sim 500 \mathrm{~m}$ at Yucca Mountain).
Alternatively, for climate variations with a time period of $T \sim 10^{5} \mathrm{yr}\left(\sim 3 \times 10^{12} \mathrm{~s}\right)$, we have from Eq. [17] and [18] an estimate of

$\delta_{c} \sim(1-2) \times 10^{3} \mathrm{~m}$

which is considerably larger than $L$. Consequently, during time periods of significant climatic variations, the infiltration-velocity field may be considered statistically homogeneous in space, with the parameters of the system (for example, saturation) changing adiabatically slowly in response to changes in climatic forcing.

\section{Random Advection in a Self-Similar Velocity Field}

Now we proceed to describe solute transport due to random advection in a stationary self-similar velocity field (Dykhne, 2003; Dykhne et al., 2005) with characteristics described above. For conservative solutes, we have the mass balance equation

$\frac{\partial c}{\partial t}+\nabla(\mathbf{v} c)=0$

where $c(\mathbf{r}, t)$ is the solute concentration. The volumetric moisture flux $\mathbf{v}(\mathbf{r})$ is a random function of coordinates. The velocity field satisfies the incompressibility equation

$\operatorname{div} \mathbf{v}=0$

which is valid due to the following reasoning. Here, we consider moisture flow before averaging across an ensemble of realizations. Since the flow is stationary due to mass conservation, the condition $\operatorname{div} \rho \mathbf{v}=0$ is valid, where $\rho=\rho_{1}$ in regions filled with moisture and $\rho=0$ outside of these regions. The variable $\rho_{1}$ is the density of the incompressible liquid and so can be taken out of the divergence operator. From here the incompressibility condition Eq. [22] follows. The problem has an initial condition $c(\mathbf{r}, 0)=c_{0}(\mathbf{r})$.

We solve the problem in two stages:

1. In the first stage, we assume the correlation length $\xi$ determined by Eq. [1] to be infinite.

2. Then we take into account that $\xi$ is finite.

From Eq. [3] it follows that an increase in $\xi$ should lead to a decrease in the mean advection velocity $\mathbf{u}=\langle\mathbf{v}(\mathbf{r})\rangle$. This is valid, however, only if the system size is greater than the correlation length, $L>\xi$. Real geologic media always have a finite thickness; therefore, as has been mentioned above, beyond a certain saturation the system size $L$ plays the role of $\xi$, with the mean velocity remaining finite. Nevertheless, due to $V>>u$ (see Eq. [9]), a considerable time interval exists within which the effect of a mean velocity may be neglected (see Eq. [47], below). To describe the system behavior in this interval, we take $\langle\mathbf{v}(\mathbf{r})\rangle=0$ at the present stage of the study.

Within the range $\left|\mathbf{r}_{i}-\mathbf{r}_{j}\right|>>a$ ( $a$ is the lower limit of the interval of fractality) there are no length parameters on which the problem variables could depend. Thus we assert that the velocity field of the infiltration flux is self-similar and that the $n$-point velocity correlation function, defined by the equation

$$
K_{i_{1} i_{2} \ldots i_{n}}^{(n)}\left(\mathbf{r}_{1}, \mathbf{r}_{2}, \ldots, \mathbf{r}_{n}\right)=\left\langle v_{i_{1}}\left(\mathbf{r}_{1}\right) v_{i_{2}}\left(\mathbf{r}_{2}\right) \ldots v_{i_{n}}\left(\mathbf{r}_{n}\right)\right\rangle
$$


is a uniform function of order $-n h$ at $\left|\mathbf{r}_{i}-\mathbf{r}_{j}\right|>>a$ (for all pairs of $\left.\mathbf{r}_{i}, \mathbf{r}_{j}\right)$ :

$$
\begin{aligned}
& K_{i_{1} i_{2} \ldots i_{n}}^{(n)}\left(\lambda \mathbf{r}_{1}, \lambda \mathbf{r}_{2}, \ldots, \lambda \mathbf{r}_{n}\right) \\
& =\lambda^{-n h} K_{i_{1} i_{2} \ldots i_{n}}^{(n)}\left(\mathbf{r}_{1}, \mathbf{r}_{2}, \ldots, \mathbf{r}_{n}\right)
\end{aligned}
$$

In particular, the pair correlation function has the property of Eq. [5] (or Eq. [14] in Fourier representation).

The quantity of interest is the solute concentration averaged across an ensemble of medium realizations, $\bar{c}(\mathbf{r}, t) \equiv\langle c(\mathbf{r}, t)\rangle$. It satisfies a standard macroscopic mass conservation equation:

$$
\frac{\partial \bar{c}}{\partial t}+\operatorname{div} \mathbf{q}=0
$$

where $\mathbf{q}(\mathbf{r}, t)$ is the macroscopic flux density that meets an obvious requirement: to be zero in case of a uniform concentration distribution as we consider the case with zero mean velocity. Due to linearity of the problem, and taking into account that in general the flux at a given point in space may depend on the medium state at all other points at earlier time (causality principle), we have

$q_{i}(\mathbf{r}, t)=-\int_{-\infty}^{t} \mathrm{~d} t^{\prime} \int \mathrm{d} \mathbf{r}^{\prime} f_{i j}\left(\mathbf{r}^{\prime}, t^{\prime}\right) \frac{\partial \bar{c}\left(\mathbf{r}-\mathbf{r}^{\prime}, t-t^{\prime}\right)}{\partial r_{j}}$

in which the response tensor function $f_{i j}(\mathbf{r}, t)$ is determined by the advection-velocity distribution.

Within the model under consideration (see Eq. [24]) there is no space scale to characterize system behavior at $r>>$. This allows us to take advantage of the ideas of critical phenomena theory (Ma, 1976) by considering transport processes at distances $r>>a$ to be scale invariant. In other words, we shall consider the macroscopic transport equation to be invariant with respect to the transformation

$\mathbf{r} \rightarrow s \mathbf{r}$

with all quantities of Eq. [25] and [26] transforming as

$$
B \rightarrow s^{-\Delta_{B}} B
$$

where the exponent $\Delta_{B}$ is termed the scaling dimension of the quantity $B$. The scaling dimensions of the velocity and concentration follow from Eq. [24] and the property of particle number conservation:

$$
\Delta_{v}=h, \quad \Delta_{c}=3
$$

Equations [25] and [26] make it possible to establish a relation between the time $t$ and the flux density $q$ scaling dimensions:

$\Delta_{t}=2-\Delta_{q}$

With Eq. [29] and [30] taken into account, the identity $\mathbf{q}=\langle(\mathbf{v} c)\rangle$ results in the expressions

$$
\begin{aligned}
& \Delta_{q}=h+3 \\
& \Delta_{t}=-(1+b)
\end{aligned}
$$

Using Eq. [26], [31], and [32], one can also obtain

$$
\Delta_{f}=2 h+3
$$

Note that scaling Eq. [31-33] is valid only for $h<1$. For $h>1$, classical scaling takes place (for example, $\Delta_{t}=-2$ ) because in that case tracer transport is determined by the short-range velocity distribution for which the scale invariance does not hold. Note also that the value of $\Delta_{t}$ for $h<1$ may be considered a consequence of the relation $t \sim r / v$ and the equality $\Delta_{v}=h$ resulting from Eq. [24].

In general, the value of $h$ is determined by characteristics of the ensemble of fracture systems, such as spatial distribution of fractures, fracture lengths, and fractal properties of fracture boundaries.

The exponents in Eq. [31-33] allow the main characteristics of solute transport to be determined for three different ranges of the exponent $h$.

\section{Velocity Scaling Dimension $h>1$}

Solute transport corresponds to classical diffusion with diffusivity $D \sim V a$.

\section{Velocity Scaling Dimension $h<1$}

At times sufficiently large so that the solute plume considerably exceeds its initial size, the average ensemble concentration from Eq. [25] and [26] can be written in the form (see Dykhne et al., 2005, for more details)

$\bar{c}(\mathbf{r}, t)=N \int_{b-i \infty}^{b+i \infty} \frac{\mathrm{d} p}{2 \pi i} \int \frac{\mathrm{d}^{3} k}{(2 \pi)^{3}} \frac{\exp (i \mathbf{k r}+p t)}{p[1+\varphi(\eta)]}$,

$\operatorname{Re} b>0$

where $N$ is the total solute inventory and $\eta$ is a self-similar variable:

$\eta=k^{2}\left(\frac{p}{V a^{b}}\right)^{-2 /(1+b)}$

The function $p \varphi(\eta)$ is a Fourier-Laplace transform of the response tensor function $f_{i j}(\mathbf{r}, t)$, which is determined by the random velocity field. The quantity $\varphi(\eta)$ has the following properties (Dykhne et al., 2005):

$$
\begin{array}{ll}
\varphi(\eta)=\sum_{n=1}^{\infty} a_{n} \eta^{n} \quad \text { at } \eta<<1 \\
\varphi(\eta) \sim \eta^{(1+h) / 2} & \text { at } \eta>>1
\end{array}
$$

Note that the self-similar variable $\eta$ depends on the value of the time scaling index (see Eq. [32]).

It follows from Eq. [34] that the average concentration may be represented as

$$
\bar{c}(\mathbf{r}, t)=\frac{N}{R^{3}(t)} \Phi(\zeta)
$$

with $\Phi(0) \sim 1, \Phi(\zeta) \rightarrow 0$ for $\zeta \rightarrow \infty$ and

$$
\zeta=\frac{r}{R(t)}
$$


$R(t)=\left(a^{b} V t\right)^{\gamma}, \quad \gamma=\frac{1}{1+b}$

determines the spatial extent of the solute plume at time $t$. Since $\gamma>1 / 2$ for $h<1$, the transport regime for this condition corresponds to the super-diffusion mode.

The asymptotic behavior of concentration at large distances is

$\bar{c}(\mathbf{r}, t) \cong$

$N \frac{A_{1 /(1+h)}}{\left[4 \pi R^{2}(t)\right]^{3 / 2}} \zeta^{\frac{3(1-h)}{2 h}} \exp \left(-B_{1 /(1+h)} \zeta^{(1+h) / h}\right)$

at $r>>R(t)$

where

$$
\begin{aligned}
& A_{\gamma}=\frac{1}{\varphi_{0}^{\prime}} \sqrt{\frac{2}{1-\gamma}}\left(\gamma \sqrt{\left|\eta_{0}\right|}\right)^{\frac{4 \gamma-1}{2(1-\gamma)}} \\
& B_{\gamma}=\frac{1-\gamma}{\gamma}\left(\gamma \sqrt{\left|\eta_{0}\right|}\right)^{1 /(1-\gamma)}
\end{aligned}
$$

and

$\varphi_{0}^{\prime}=\left.\frac{\mathrm{d} \varphi(\eta)}{\mathrm{d} \eta}\right|_{\eta=\eta_{0}}$

The quantity $\eta_{0}$ is the solution of the equation

$1+\varphi\left(\eta_{0}\right)=0$

Note that the concentration tail in the random advection model at $h<1$ is reduced in comparison to the classical Gaussian exponential. This result is in sharp contrast to fractional diffusion models (with fractional spatial derivatives), whose tails are of the power-law type (Metzler and Klafter, 2000). Below we consider media with traps, the presence of which also leads to a change in tailing behavior (i.e., the spatial decay of tails becomes less). A discussion of the final form of solute concentration tails is given below.

\section{Velocity Scaling Dimension $h=1$}

Solute transport in this case is a logarithmically modified classical diffusion process. The ensemble-averaged concentration is described by the expression

$\bar{c}(\mathbf{r}, t) \cong \frac{N}{[4 \pi R(t)]^{3 / 2}} \exp \left\{-\left[\frac{r}{R(t)}\right]^{2}\right\}$

with

$R(t)=\sqrt{\tilde{D} t} \ln ^{1 / 4}\left(\frac{\sqrt{\tilde{D} t}}{a}\right), \quad \tilde{D} \sim V a$

Now we address the case where the advection-velocity field has a finite correlation length $\xi$ (Kondratenko and Matveev, $2007)$ for $h<1$. For this condition, the advection velocity may be represented in the form

$\mathbf{v}(\mathbf{r})=\mathbf{u}+\mathbf{v}^{\prime}(\mathbf{r})$

where the correlation function of the "random" term $\mathbf{v}^{\prime}(\mathbf{r})$ possesses the properties of Eq. [24], which are now valid only at $\left|\mathbf{r}_{i}-\mathbf{r}_{j}\right|<\xi$. All correlations decay at $\left|\mathbf{r}_{i}-\mathbf{r}_{j}\right|>\xi$ exponentially fast. The average velocity is determined by the estimate of Eq. [3].

At short times, $t<t_{*}$, where

$t_{*}=\frac{\xi}{u} \sim \frac{\xi^{1+h}}{a^{h} V}$

the results expressed by Eq. [37-40] remain valid. At long times, $t>t_{*}$, the classical diffusive regime is realized:

$\bar{c}(\vec{r}, t)=\frac{N}{\left(4 \pi D_{\text {eff }} t\right)^{3 / 2}} \exp \left[-\frac{(\mathbf{r}-\mathbf{u} t)^{2}}{4 D_{\text {eff }} t}\right]$

with $D_{\text {eff }} \sim u \xi$

This expression holds true for the condition $|\mathbf{r}-\mathbf{u} t|<<$ t. At large distances with $|\mathbf{r}-\mathbf{u} t|>>u t$, the concentration behavior is described by the asymptotic expression Eq. [40], which also provides the concentration asymptotics at short times for $h<1$. Therefore, in the case of a finite correlation length, $\xi<\infty$, the concentration tail at $t>t_{*}$ has a two-stage structure. The near stage is the classical one, while the far stage corresponds to superdiffusive asymptotics. The transition between the two stages of asymptotics occurs when

$\bar{c} \propto \exp \left(-A \frac{t}{t_{*}}\right) \quad$ with $A \sim 1$

\section{Solute Transport in Fractal Dual-Porosity Media}

We next consider transport processes in terms of solute concentrations in a highly permeable medium (which is a network of channels constituting the backbone of the percolation cluster). These concentrations are named active concentrations, or simply concentrations. A matrix and a set of wetted "dead-end" fractures (fractures in which water is motionless) play the role of traps or a low-permeability (passive) medium. Dynamic fluctuations also provide a mechanism for loss of active particles in traps. Two points are important in our consideration: (i) the geometry of the conducting medium is fractal; and (ii) random advection is the main mechanism of particle transport.

Based on the results obtained by Dykhne (2004b) and Dykhne et al. (2008), the presence of traps formally can be described by the random advection model by adding a trap term to the microscopic mass conservation Eq. [25]:

$$
\partial c / \partial t+\Phi+\operatorname{div} \mathbf{q}=0
$$

where $\Phi(\mathbf{r}, t)$ is the density of traps defined by the expression

$$
\Phi(\mathbf{r}, t)=\int_{-\infty}^{t} \varphi\left(t-t^{\prime}\right) c\left(\mathbf{r}, t^{\prime}\right) \mathrm{d} t^{\prime}
$$

with an inverse-power kernel. In turn, it is equivalent to the following replacement in Eq. [34]:

$$
p \rightarrow p \pi(p)
$$

where $p \pi(p)$ is the Laplace transform of the kernel $\varphi(t)$ from Eq. [51] and is determined by

$$
\pi(p) \sim\left(p t_{n}\right)^{z_{n}-1} \quad \text { at } t_{n+1}^{-1}<p<t_{n}^{-1}
$$


describing the predominant type of trap within each time interval $\left[t_{n}, t_{n+1}\right]$. Note that usually $t_{n}<<t_{n+1}$ and $z_{1}>z_{2}>z_{3} \ldots$. After replacement of Eq. [52], Eq. [34] takes the form

$\bar{c}(\mathbf{r}, t)=$

$N_{0} \frac{1}{2 \pi i} \int_{b-i \infty}^{b+i \infty} \frac{\mathrm{d} p}{p} \int \frac{\mathrm{d}^{3} k}{(2 \pi)^{3}} \frac{\exp (i \mathbf{k r}+p t)}{\pi(p)[1+\varphi(\eta)]}$

$\operatorname{Re} b>0$

In the presence of traps, the total active solute inventory

$N(t)=\int \mathrm{d}^{3} r \bar{c}(\mathbf{r}, t)$

becomes time dependent. Substituting Eq. [54] into [55], we have

$N(t)=N_{0} \frac{1}{2 \pi i} \int_{b-i \infty}^{b+i \infty} \frac{\mathrm{d} p}{p \pi(p)} \exp (p t), \quad \operatorname{Re} b>0$

where $N_{0}=N(0)$. The integration in Eq. [56] results in an expression for the total active solute inventory:

$N(t)=N_{n}(t)=N_{0}\left(\frac{t_{n}}{t}\right)^{1-z_{n}} \Gamma\left(z_{n}\right)$

at $t_{n-1}<t<t_{n}$

where $\Gamma(z)$ is the gamma function.

Taking into account Eq. [56], we find from Eq. [54] the structure of the active solute concentration [at $\left.r \leq R_{n}(t)\right]$ in the time interval $t_{n-1}<t<t_{n}$ :

$\bar{c}(\mathbf{r}, t) \cong \frac{N_{n}(t)}{R_{n}^{3}(t)} \Psi\left(\zeta_{n}\right)$

where $N_{n}(t)$ is the total active solute inventory at time $t$,

$\zeta_{n}=\frac{r}{R_{n}(t)}$

is a dimensionless self-similar variable, and

$R_{n}(t)=\left(a^{h} V t^{z_{n}} t_{n}^{1-z_{n}}\right)^{1 /(1+h)}$

is the solute plume size at $t_{n-1}<t<t_{n}$. Note that

$R_{n}(t) \propto t^{\gamma_{n}} \quad$ with $\gamma_{n}=\frac{z_{n}}{1+h} \quad$ at $\quad t_{n-1}<t<t_{n}$

The function $\Psi(\zeta)$ possesses the following properties:

$\Psi(0) \sim 1, \quad \Psi\left(\zeta_{n}\right)<<1 \quad$ at $\zeta_{n}>>1$

The asymptotic behavior of the concentration at large distances can be obtained from Eq. [54]:

$$
\begin{aligned}
& \bar{c}(\mathbf{r}, t) \cong \frac{N_{m}(t)}{\left[4 \pi R_{m}^{2}(t)\right]^{3 / 2}} A_{\gamma_{m}}\left(\gamma_{m} \sqrt{\left|\eta_{0}\right|}\right)^{\frac{1-z_{m}}{1-\gamma_{m}}} \\
& \Gamma\left(z_{m}\right) \zeta_{m}^{\frac{6 \gamma_{m}-2 z_{m}-1}{2\left(1-\gamma_{m}\right)}} \exp \left[-B_{\gamma_{m}} \zeta_{m}^{1 /\left(1-\gamma_{m}\right)}\right]
\end{aligned}
$$

at $t_{n-1}<t<t_{n}$

where the constants $A_{\gamma}$ and $B_{\gamma}$ are of the order of unity and are determined by Eq. [41]. The value of index $m$ is determined by the solution of $\gamma_{m} \zeta_{m} \sqrt{\left|\eta_{0}\right|}=(p t)^{1-\gamma_{m}}$

in the interval $t_{m}{ }^{-1}<p<t_{m-1}{ }^{-1}$. For moderately large $\zeta=r / R(t)$, we have $m=n$, while the asymptotics of the concentration are determined by the same regime as the main plume concentration. As the value of $\zeta$ increases (see Eq. [64] and [53]), however, earlier transport regimes determine the concentration asymptotics. In other words, as the distance from the source increases (at a fixed time), the regimes of solute transport are reproduced by the shape of concentration asymptotics in an inverse time order. For illustration, the solute plume size (Eq. [60]) and concentration asymptotics of the tail (Eq. [63]) are shown schematically in Fig. 1 and 2.

$\mathrm{R}(\mathrm{m})$

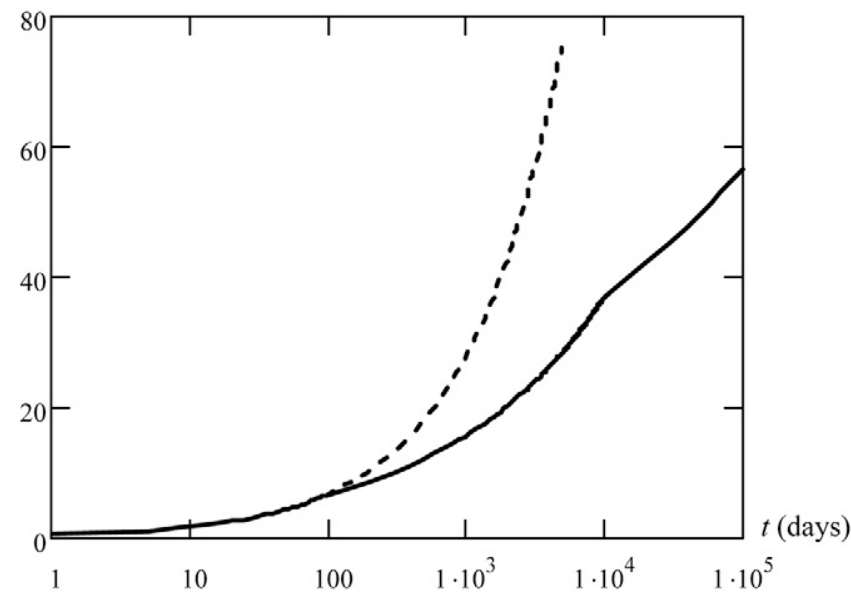

FIG. 1. Dependence of solute plume size $(R)$ on time $(t)$. The solid line includes the influence of sinks, while the dashed line ignores sinks. The following parameter values were used for the calculation: $a=10^{-1} \mathrm{~m}, V=10^{-5} \mathrm{~m} \mathrm{~s}^{-1}, h=0.6, \alpha=1$ for $t<10^{2} \mathrm{~d}, \alpha=0.6$ for $10^{2}<t<10^{4} \mathrm{~d}, \alpha=0.3$ for $t>10^{4} \mathrm{~d}$.

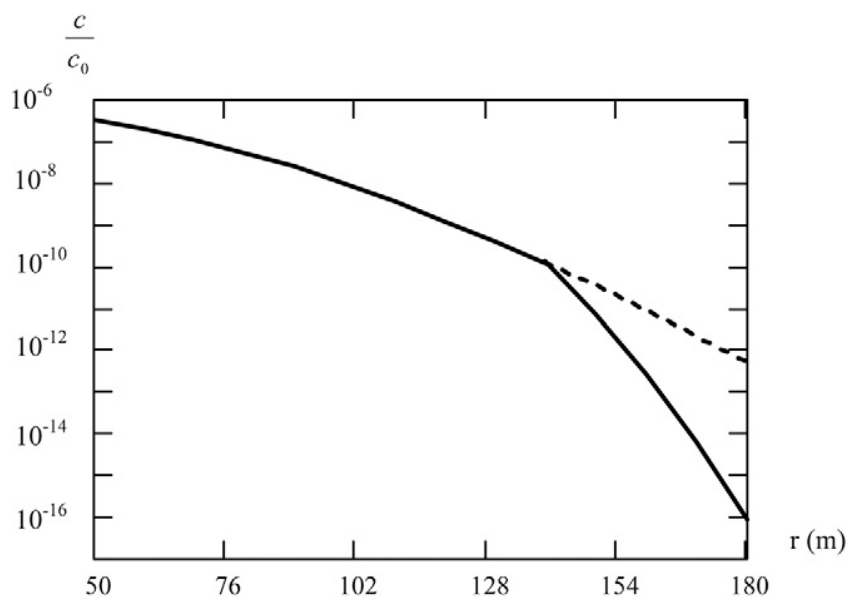

FIG. 2. Spatial dependence of the concentration tail asymptotics; $c / c_{0}$ is the ratio of the concentration at distance $r$ from the source to that at the source site. The calculation was made for time $t=$ $10^{3} \mathrm{~d}$. The dashed line shows the concentration behavior when the change of transport regime at earlier times is neglected. The following parameter values were used for the calculation: $a=10^{-1} \mathrm{~m}$, $V=10^{-5} \mathrm{~m} \mathrm{~s}^{-1}, h=0.6, \alpha=0.6$. 


\section{Renormalization of Solute Source Strength Due to Fluctuation Effects}

According to the previous analysis, the master equation for the active solute concentration may be represented in the form of Eq. [50], where $\mathbf{q}(\mathbf{r}, t)$ is the macroscopic flux density determined by Eq. [26] and $\Phi(\mathbf{r}, t)$ is the density of traps defined by Eq. [51].

The macroscopic Eq. [50] is valid within the fractality interval $a<r<\xi$. For the problems discussed above, the size of the solute source (initial plume size) $r_{0}$ was considered to also fall in the range $a<r_{0}<<\xi$. To describe solute transport for the condition $r_{0}<a$, strong fluctuations in the medium properties at the scales $r_{0}<r<a$ should be taken into account. We now consider this problem.

Assume that time is sufficiently large so that $R(t)>>a$. Let us surround the solute source with a closed surface $S_{1}$ having a characteristic radius $R_{1} \gg$ a. A total macroscopic solute flux $Q$ through $S_{1}$ may be represented as

$Q=K Q_{0}$

where $Q_{0}$ corresponds to the source strength (i.e., the total solute flux) for a fixed concentration at the source boundary in the absence of medium property fluctuations, and $K$ is the power renormalizing factor.

The factor $K$ cannot be derived from macroscopic considerations because it requires knowledge of the medium characteristics at $r<a$, where they strongly fluctuate. This situation resembles the problem of tunneling barrier conductivity explored by Raikh and Ruzin (1991). Here we take advantage of their approach. Like the conductivity in Raikh and Ruzin (1991), the factor $K$ in our problem is determined by rare combinations of favorable conditions_- "leakage paths." Following Raikh and Ruzin (1991), we will call them punctures. The contribution $F$ to the transmission coefficient from an individual puncture is statistically distributed across a wide range of values. This contribution can be expressed as $F=F_{0} \exp (-u)$, where $u$ is a stochastic variable uniformly distributed across the interval from 0 to $\infty$. The distribution of puncture concentrations per unit area of the source boundary can be expressed as

$$
\rho(u)=\left(S_{0}\right)^{-1} \exp [-\Omega(u)]
$$

where $S_{0}$ is the characteristic cross-sectional size of the puncture, which is small compared with the average distance between punctures, and $\Omega(u)$ is a function having the properties $\Omega(u)$ >> $1, \partial \Omega / \partial u<0$, and $\partial^{2} \Omega / \partial u^{2}>0$.

The analysis using an averaging procedure across the puncture concentration distribution of Eq. [64] leads to the following results. For large source sizes, the renormalizing factor is close to unity:

$K \cong 1$ at $S>>S_{*}$

where $S$ is the source surface area, $S_{*}$ is given by the expression

$$
S_{*}=S_{0} \exp \left[\Omega\left(u_{\mathrm{opt}}\right)\right]
$$

and the value $u_{\mathrm{opt}}$ is determined by the equation

$$
[\partial \Omega(u) / \partial u]_{u=u_{\mathrm{opt}}}+1=0
$$

For small source sizes, the renormalizing factor decreases with $S$ :

$$
K \propto \exp \left[-\left(u_{f}-u_{\mathrm{opt}}\right)\right] \text { at } S<<S_{*}
$$

with $u_{f}$ determined by

$$
\frac{S}{S_{*}} \exp \left[\Omega\left(u_{\mathrm{opt}}\right)-\Omega\left(u_{f}\right)\right]=1
$$

Note that we have $K<<1$ at $S<<S_{*}$.

One additional effect caused by the fluctuations concerns the statistical scatter of the renormalization factor $K$. The relative scatter $\Delta(K) \equiv\left\langle(K-\langle K\rangle)^{2}\right\rangle /\langle K\rangle$ is small for large source sizes and becomes large for small source sizes:

$\Delta(K)<<1$ at $S>>S_{*}, \quad \Delta(K)>1$ at $S<<S_{*}$

\section{Conclusions}

We developed new elements to generalize the dual-porosity model for moisture infiltration and solute transport in unsaturated rocks, taking into account fractal aspects of the percolation process.

Infiltration processes in unsaturated fractured rocks show self-organized criticality that leads to a fractal spatial structure of the flow paths. Percolation theory relations were applied to the moisture seepage problem for this case. We established that for scales $r<<\xi$, where $\xi$ is the correlation length, the random component of the seepage velocity is considerably greater than the average velocity. The two become comparable only at scales $r \sim \xi$.

In addition to stationary (spatial) fluctuations of the advection-velocity field, dynamic (time-dependent) fluctuations arise in the system. They possess a self-similarity property and are akin to $1 / f$ (flicker) noise.

The effect of variations in the precipitation intensity on seepage processes in the geologic media considered here depends on their time scale. Seasonal variations in the moisture inflow manifest themselves only in a relatively thin boundary layer. In contrast, slow climatic variations penetrate deeply into the medium, resulting in adiabatically slow variations in medium characteristics.

Within the framework of the fractal generalization of the double-porosity model, we showed that the advective solute transport regime in the considered media is determined by a competition between two mechanisms. A superdiffusive transport regime arises in the case of slow power-like decay of flow-velocity correlations. The presence of a sharp contrast in medium properties on the other hand gives rise to slower solute transport, including subdiffusion mode. The concentration tails in both anomalous transport regimes (super-and subdiffusion) are of the exponential type. Compared with classical Gaussian tails, they correspond to contracted and stretched exponential tails for super- and subdiffusion modes, respectively. Here it should be noted that, from our model, it follows that tails less severe than Gaussian tails occur only when the transport regime is superdiffusive. When traps are present in the medium (a rather common case in the problem of transport in geologic media), the regime of transport may become subdiffusive (Eq. [60]), in which case 
the tails become more pronounced than the Gaussian exponential tails (Eq. [63]) but are still exponential. No power-law tails occur, in stark contrast to those typical of fractional derivative and continuous-time random walk models.

The change in transport regimes with time results in a multistage structure of the concentration tails. With increasing distance from the source at a fixed time, concentration asymptotics reproduce the transport regimes in inverse time order.

Spatial fluctuations in medium properties lead to a renormalization of the solute source strength for small source sizes. In addition, the renormalizing factor is subject to a significant statistical scatter.

\section{ACKNOWLEDGMENTS}

We acknowledge support from the U.S. Department of Energy (USDOE) under the U.S. Civilian Research and Development Foundation Grant Assistance Program Project RG0-20101-RW40 with the Institute of Nuclear Energy Safety (IBRAE) of the Russian Academy of Sciences, and from the Russian Foundation for Basic Research (RFBR) under Project 06-08-00176a. Dr. Pruess also acknowledges support from the USDOE Office of Civilian Radioactive Waste Management under Contract Number DE-AC02-05CH11231.

\section{References}

Bak, P., C. Tang, and K. Wiesenfeld. 1987. Self-organized criticality: An explanation of 1/fnoise. Phys. Rev. Lett. 59:381-384.

Bak, P., C. Tang, and K. Wiesenfeld. 1988. Self-organized criticality. Phys. Rev. A 38:364-374

Barenblatt, G.I., I.P. Zheltov, and I.N. Kochina. 1960. Basic concepts in the theory of seepage of homogenous liquids in fissured rocks. J. Appl. Math. Mech. 24:1225-1303.

ben-Avraham, D., and S. Havlin. 2000. Diffusion and reactions in fractals and disordered systems. Cambridge Univ. Press, Cambridge, UK.

Berkowitz, B., and C. Braester. 1991. Dispersion in sub-representative elementary volume fracture network: Percolation theory and random walk approaches. Water Resour. Res. 27:3159-3164.

Bolshov, L.A., P.S. Kondratenko, K. Pruess, and V.N. Semenov. 2008. Nonclassical transport processes in geologic media. Review of field and laboratory observations and basic physical concepts. Vadose Zone J. 7:1135-1144 (this issue).

Chang, J., and Y.C. Yortsos. 1990. Pressure-transient analysis of fractal reservoirs. SPE Form. Eval. 5(1):31-38.

Dullien, F.A.L. 1979. Porous media: Fluid transport and pore structure. Academic Press, San Diego, CA.

Dykhne, A.M. 2003. Interdisciplinary fundamental research to further develop the methods of describing and modeling contaminant transport processes in unsaturated rocks. Phase I, Product 2.4. IBRAE RAN, Moscow.

Dykhne, A.M. 2004a. Interdisciplinary fundamental research to further develop the methods of describing and modeling contaminant transport processes in unsaturated rocks. Phase II, Product 2. IBRAE RAN, Moscow.

Dykhne, A.M. 2004b. Interdisciplinary fundamental research to further develop the methods of describing and modeling contaminant transport processes in unsaturated rocks. Phase I, Product 2.5. IBRAE RAN, Moscow.

Dykhne, A.M., I.L. Dranikov, P.S. Kondratenko, and L.V. Matveev. 2005. Anomalous diffusion in a self-similar random advection field. Phys. Rev. E 72:061104.

Dykhne, A.M., I.L. Dranikov, P.S. Kondratenko, and L.V. Matveev. 2008. Transport regimes and concentration tails for classical diffusion in heterogeneous media with sharply contrasting properties. Vadose Zone J. 7:1145-1151 (this issue).

Gerke, H.H., and M.Th. van Genuchten. 1993. A dual-porosity model for simulating the preferential movement of water and solutes in structured porous media. Water Resour. Res. 29:305-319.

Hunt, A.G. 2004a. Percolative transport in fractal porous media. Chaos Solitons Fractals 19:309-325.

Hunt, A.G. 2004b. Comparing van Genuchten and percolation theoretical formulations of the hydraulic properties of unsaturated media. Vadose Zone J. 3:1483-1488.
9 Hunt, A.G. 2004c. Continuum percolation theory for pressure saturation characteristics of fractal soils: Extension to non-equilibrium. Adv. Water Resour. 27:245-257.

Isichenko, M.B. 1992. Percolation, statistical topography and transport in random media. Rev. Mod. Phys. 64:961-1043.

Kondratenko, P.S., and L.V. Matveev. 2007. Random advection in a fractal medium with finite correlation length. Phys. Rev. E 75:051102, doi:10.1103/ PhysRevE.75.051102.

Ma, S.-K. 1976. Modern theory of critical phenomena. Benjamin, London.

Metzler, R., and J. Klafter. 2000. The random walks guide to anomalous diffusion: A fractional dynamics approach. Phys. Rep. 339:1-77.

Pruess, K. 1999. A mechanistic model for water seepage through thick unsaturated zones in fractured rocks of low permeability. Water Resour. Res. 35:1039-1051.

Raikh, E.M., and I.M. Ruzin. 1991. Transmittancy fluctuations in randomly non-uniform barriers and incoherent mesoscopics. p. 315-368. In B.L. Altshuler et al. (ed.) Mesoscopic phenomena in solids. Elsevier Science, Amsterdam.

Ray, C., T.R. Ellsworth, A.J. Valocchi, and C.W. Boast. 1997. An improved dual porosity model for chemical transport in macroporous soils. J. Hydrol. 193:270-292.

Sahimi, M. 1993. Flow phenomena in rocks: From continuum models to fractals, percolation, cellular automata, and simulated annealing. Rev. Mod. Phys. 65:1393-1534.

Sahimi, M., and A.O. Imdakh. 1998. The effects of morphological disorder on hydrodynamic dispersion in flows through porous media. J. Phys. A: Math. Gen. 21:3833-3870.

Vespignani, A., and S. Zapperi. 1998. How self-organized criticality works: A unified mean-field picture. Phys. Rev. E 57:6345-6362. 